\title{
Carnets
}

Revue électronique d'études françaises de l'APEF

Première Série - 2 Numéro Spécial 10-11 | 2011

D'un Nobel l'autre

\section{Introduction à la lecture de Jean-Marc Lovay: à propos du livre Epître aux Martiens}

\section{Maria Hermínia Amado Laurel}

\section{(2) OpenEdition}

Journals

Édition électronique

URL : http://journals.openedition.org/carnets/5518

DOI : 10.4000/carnets. 5518

ISSN : 1646-7698

Éditeur

APEF

Édition imprimée

Date de publication : 1 janvier 2011

Pagination : 81-98

Référence électronique

Maria Hermínia Amado Laurel, «Introduction à la lecture de Jean-Marc Lovay: à propos du livre Epitre aux Martiens », Carnets [En ligne], Première Série - 2 Numéro Spécial 10-11| 2011, mis en ligne le 16 juin 2018, consulté le 02 mai 2019. URL : http://journals.openedition.org/carnets/5518 ; DOI : $10.4000 /$ carnets. 5518

\section{(c) (1) (8)}

Carnets est mis à disposition selon les termes de la licence Creative Commons - Atribution - Pas d'utilisation commerciale 4.0 International. 


\title{
INTRODUCTION À LA LECTURE DE JEAN-MARC LOVAY À propos du livre Épître aux Martiens
}

\author{
MARIa HermínIa Amado LAUREL \\ Universidade de Aveiro \\ hlaurel@ua.pt
}

\begin{abstract}
Résumé
Composé en 1967 par Jean-Marc Lovay, le roman Épître aux Martiens n'a été publié qu'en 2004, aux Editions Zoé. Paru lorsque des titres aussi importants que La Tentation de l'Orient (1970), Polenta (1980), ou Aucun de mes os ne sera troué pour servir de flûte enchantée (1998) avaient déjà assuré la notoriété de l'auteur, ce livre constitue une initiation indispensable à un auteur difficile, mais alerte sur le monde contemporain. Notre analyse portera sur ce roman, que nous situerons dans le contexte de ses origines, mais dont la lecture, déjouant le lecteur inaverti, convoque celle d'autres grands auteurs romands de la modernité, dont Maurice Chappaz, dans la poursuite d'un travail inépuisable sur la langue littéraire.
\end{abstract}

\section{Abstract}

Althought it was written in 1967, Jean-Marc Lovay's novel Épitre aux Martiens was published in 2004 (Éditons Zoé). Published when so important books as La Tentation de l'Orient (1970), Polenta (1980), or Aucun de mes os ne sera troué pour servir de flûte enchantée (1998) had already insured their author's fame, this novel reveals itself as an important introduction to a difficult author, but much aware of contemporary word. Our analysis will focus the origins of this novel; reading Lovay's evoques the work on French Language pursued by other important Swiss writers, as Maurice Chappaz.

Mots-clés: Jean-Marc Lovay, Maurice Chappaz, écriture littéraire, catastrophe, condition humaine Keywords: Jean-Marc Lovay, Maurice Chappaz, literary writing, catastrophe, human condition 
Épitre aux Martiens est le premier roman d'une liste longue de plus de vingt titres que l'écrivain suisse Jean-Marc Lovay a écrits depuis l'âge de 19 ans. Composé en 1967, couronné par le prestigieux prix Georges Nicole deux ans après, ce roman n'a pourtant vu le jour qu'en 2004, aux Editions Zoé. Jean-Louis Kuffer l'a bien remarqué lors de la parution de ce livre en librairie, en constatant que ce roman portait les traces de l'époque où il avait été rédigé, et se donnait en partie à lire comme un témoignage particulier de la contre-culture qui inspirait la jeunesse contemporaine. Paru lorsque des titres aussi importants que La Tentation de l'Orient (1970), Polenta (1980), ou Aucun de mes os ne sera troué pour servir de flûte enchantée (1998) avaient déjà assuré la notoriété de l'auteur, ce livre constitue pourtant une initiation indispensable à l'univers délirant, éventuellement parsemé d'humour, d'un auteur difficile mais sans doute alerte sur la condition humaine, dont l'œuvre évoque tantôt des scénarios de science-fiction que des espaces préfigurant des catastrophes imminentes ou qui en constituent des vestiges. Notre analyse portera sur ce roman, que nous situerons dans le contexte de ses origines, mais dont la lecture, déjouant déjà le lecteur inaverti, convoque celle d'autres grands auteurs romands de la modernité, tels que Maurice Chappaz ou Blaise Cendrars, dans la poursuite d'un travail inépuisable sur la langue littéraire.

La lecture du livre Épître aux Martiens nous convie à une méthodologie peu commune: celle de la lecture de l'œuvre d'un écrivain, à rebours. Et ceci dû à une circonstance d'ordre purement éditorial: bien que composée en 1967, l'Épître aux Martiens ne fut publiée que trente sept ans plus tard.

L'histoire quelque peu romanesque de ce livre est rappelée par son auteur, qui constate la disparition du "tapuscrit envoyé au concours" pour le prix référé, dans la préface qu'il a rédigée en l'automne de 2003 pour sa publication aux Éditions Zoé. Intitulée "Disparition et réapparition du tapuscrit d'un jeune auteur", cette préface ne manque pas d'en rajouter au mystère dont s'entoure I'histoire du livre: après être entrés dans un long et "secret sommeil des malles ou des cartons entreposés dans des galetas ou des caves", quelques manuscrits du livre auraient vu le jour, dont celui de la "toute première version originale de l'Épître aux Martiens ainsi qu'une copie de la version corrigée au Népal"1 , que l'auteur "déniche" lui-même, "en automne 2001 [...] dans une cave entre un vieil aspirateur et un pied de sapin de Noël" (Lovay, 2004: 7).

Au moment où ce livre paraît, au printemps de 2004, Lovay est déjà un auteur reconnu par la critique: distingué à l'âge de 19 ans pour son premier manuscrit, ayant publié ensuite deux romans chez Gallimard ${ }^{2}$, des récits, des conférences, la correspondance avec

\footnotetext{
${ }^{1}$ Nous reviendrons plus loin sur le voyage de Lovay en Asie à la fin des années 1960.

2 Les régions céréalières (1976) et Le Baluchon maudit (1979). Lovay se fera publier plus tard chez l'éditeur genevois Zoé.
} 
Maurice Chappaz entre 1968-69 et des entretiens s'ensuivront. Son dernier roman, Tout làbas avec Capolino, a intégré la liste des auteurs candidats au Prix Wepler 2009.

La lecture d'une œuvre de jeunesse qui n'est divulguée qu'à un moment avancé de la carrière d'un auteur pose plusieurs problèmes.

Qu'on le veuille ou non, cette lecture sera forcément conditionnée par l'image que le lecteur s'est faite de l'auteur - image créée non seulement par la lecture de ses publications, mais aussi par la fortune critique de l'auteur, en d'autres mots, par sa réception critique, de même que par l'image que l'auteur s'est donnée de lui-même au long de toutes ces années ${ }^{3}$.

A ce propos, la lecture des extraits de presse parus lors de la publication de l'Épître aux Martiens ${ }^{4}$ peut être révélatrice. Comment a été reçue cette publication par la presse de spécialité? A-t-elle été considérée comme une introduction à l'œuvre postérieure? La critique lui a-t-elle attribué le statut d'œuvre de jeunesse?

Globalement, nous pourrions conclure que le dossier de presse réuni à cette occasion par son éditeur insiste sur deux points: primo, sur le caractère insolite de l'événement en lui-même - classé comme une 'découverte', secundo, sur la situation de ce livre dans l'œuvre de l'écrivain: "Lovay avant Lovay, est-ce déjà du Lovay?", s'interroge le journal Le Temps, quotidien de Genève, le 6 mars 2004. L'adjectivation est riche, rien qu'à lire les titres de ce dossier, pour classifier ce livre: "écriture envoûtante"5, "épître cosmique"6, "texte halluciné"7, "genèse psychédélique de l'œuvre de Lovay"8, "révélation", "création hallucinée"9; l'adjectivation se multiplie, si nous entrons dans le détail des articles. L'hebdomadaire genevois Le Courrier, dans son édition du 8 avril 2004 accentue les "fulgurances du style" de l'écrivain et la "langue qui bouleverse les repères de la raison et les catégories mentales", langue qu'il qualifie de "sorte de transe poétique ultralucide", pour créer un "monde [...] à la fois hallucinant et cohérent"; Le Nouvelliste, Feuille d'avis du Valais, à son tour, souligne d'identité d'"une écriture d'un véritable écrivain", aux "touches d'humour omniprésentes". Le dossier de presse constitué à l'occasion de la parution du livre réunit plusieurs articles qui convergent dans la consécration du style de Lovay d'après les effets qu'il produit sur les habitudes du lecteur, qu'il bouleverse. "Cette ambiguïté marque

\footnotetext{
${ }^{3}$ N'insistons pas sur cette image, pour un écrivain qui choisit de vivre en écart de la société médiatisée contemporaine.

${ }^{4}$ Nous remercions Madame le Prof. Doris Jakubec de nous avoir introduite auprès des Éditions Zoé qui nous ont aimablement faculté la lecture de leur dossier de presse sur l'auteur.

5 Theytaz, Jean-Marc, "Les méandres d'une écriture envoûtante", Le Nouvelliste, Feuille d'avis du Valais, 14.02.2004.

6 "L'épître cosmique du jeune Lovay", Le Courrier, 08.04.2004.

7 Id., ibidem.

${ }^{8}$ Jean-Louis Kuffer, "L'entrée du labyrinthe. Exhumation. Genèse psychédélique de l'œuvre de Jean-Marc Lovay, Épître aux Martiens paraît avec trente ans de retard. Perdu et retrouvé, ce texte tient de la révélation", 24 Heures, mardi, 24 février 2004.

9 "Jean-Marc Lovay ou la création hallucinée" est le titre du discours prononcé par Charles Méla, professeur de littérature médiévale à l'Université de Genève et directeur de la Fondation Bodmer, lors de l'attribution du Prix quadriennal de la Ville de Genève à l'écrivain, en juin 2003. Nous remercions les Éditions Zoé de l'envoi de ce texte, d'après l'indication de Mme Doris Jakubec.
} 
Épître aux martiens jusque dans sa substance, une langue unique, poétique au sens de l'invention, halluciné, minérale, essentielle, qui ne donne sens que dans l'abandon à un certain affolement de la lecture", pouvons-nous lire dans le journal La Liberté (samedi 28 février 2004).

Or, l'écriture de ce livre ayant précédé de neuf ans la publication du prochain roman de l'auteur, Les régions céréalières (paru en 1976, chez Gallimard, Prix Rambert), le lecteur aurait pu être mené soit à l'oublier, soit à établir une filiation possible entre les deux romans. Filiation qui lui aurait très probablement fait considérer l'Épître aux Martiens comme une première tentative d'écriture romanesque, en l'occurrence une introduction aux œuvres postérieures de l'auteur. Ce livre risquerait ainsi de se voir attribuer un statut de minorité dans l'ensemble de l'œuvre de Lovay; position qui dénoterait, d'autre part, une conception évolutive (sinon, évolutionniste) de l'œuvre d'un écrivain. L'ambiguïté de classifications telles que "œuvres de jeunesse" et "œuvres de maturité", d'ailleurs souvent utilisées pour l'établissement de chronologies d'écrivains, ne convient pourtant en aucune façon à la production d'un auteur qui faisait son entrée dans la scène littéraire par un récit tel celui de l'Épître aux Martiens.

Or, non seulement l'auteur refuse cette possibilité:

trente-six ans après avoir écrit ce texte je veux repousser une complaisante propension à trop humblement estimer naïf un premier livre de jeunesse, en avouant que je ne perçois aucune naïveté dans la trajectoire menant le héros-narrateur à la conclusion qu'il est conduit malgré lui mais aussi par sa propre détermination à suivre et à diriger l'ombre de son destin en refusant d'obéir aux ordres qui se répercutent décennie après décennie. (Lovay, 2004: 7-8)

comme chacune de ses œuvres constitue en elle-même, refusant tout essai de classification typologique, l' "ouverture" à un univers d'écriture qui ne manquera pas de surprendre le lecteur et de constituer un nouveau défi à ses capacités. Le journaliste littéraire et écrivain suisse Jean-Louis Kuffer, auteur de l'éditorial consacré à la parution de cette œuvre dans la page littéraire dont il était le responsable au journal 24 heures, le 24 février 2004, l'avoue: “On n'entre pas dans le labyrinthe de Jean-Marc Lovay comme dans un moulin. Pour [sa] part, [il y aurait] mis des années". Un univers dans lequel le lecteur aura plutôt du mal à pénétrer, si tant est qu'il puisse jamais quitter ses livres tranquillement, après quelques heures de lecture agréables, à l'image du lecteur à la "main blanche [...] qui [s'enfonçait] dans un moelleux fauteuil" que Balzac se figurait dire, en prenant un de ses livres au hasard: "peut-être cela va-t-il m’amuser" (Balzac, 1989: 22). 
L'humour, qui fait irruption dans les pages de Lovay, il faudra le reconnaître, n'a peutêtre pas pour but immédiat ni exclusif de nous amuser; il institue plutôt une corrélation entre la fiction et le réel, un continuum symbolique entre l'écriture et ses référents: "J'ai été dans la rue avec sur moi l'odeur purulente du palais ministériel. Je me suis dit que c'était l'odeur véritable, et que je me faisais des illusions, que tout cela était bien vrai, et vrai, et vital!“ (Lovay, 2004: 53).

Une forte mise en alerte est sous-jacente à cet épisode aux tonalités sarcastiques. L'effet de surprise causé par les corrélations implicites de ce passage de même que l'engagement dont elles font preuve de la part de l'auteur, à l'abri de l'humour qui les soustend, deviennent partie constitutive de textes qui se donnent à lire comme un véritable défi aux capacités de résistance du lecteur; et pourtant, il n'est pas facile d'abandonner un livre de Jean-Marc Lovay une fois qu'on en a entamé la lecture. Comment expliquer alors ce paradoxe?

Nous situerons notre lecture de l'Épître aux Martiens dans le contexte de ses origines, en ce que ce roman est la première mise en forme, par son auteur, d'une conception de l'écriture littéraire que la lecture d'autres textes ne manque pas de convoquer. Ainsi, nous nous attarderons, en particulier, sur la correspondance entretenue par Lovay avec Maurice Chappaz entre 1968 et 1969, dont la première édition parut en 1970, intitulée La Tentation de l'Orient, et sur les trois conférences que le romancier a regroupées sous le titre Conférences aux Antipodes (1987).

Les textes de Lovay instituent un pacte de lecture qui valorise tout autant le travail du romancier que celui du lecteur. Ils se présentent comme un espace privilégié de concentration dans l'écriture romanesque et la lecture, un espace hostile à toute distraction, que ce soit celle de l'écrivain ou celle du lecteur. Ces textes s'inscrivent, par cette qualité, dans le courant de toute une réflexion sur le romanesque qui caractérise la littérature en langue française à partir de la fin des années 1940, et à laquelle la question posée par JeanPaul Sartre en 1948, "Qu'est-ce que la littérature?" ne saurait être étrangère. Les nouveaux romanciers ${ }^{10} \mathrm{y}$ apporteront des éléments de réflexion incontournables. À l'instar de Nathalie Sarraute $^{11}$, pour laquelle "les drames constitués par [les] actions encore inconnues [l']intéressaient en eux-mêmes. Rien ne pouvait en distraire [son] attention. Rien ne devait en distraire celle du lecteur: ni caractères des personnages, ni intrigue romanesque à la faveur

\footnotetext{
${ }^{10}$ Le "nouveau roman" n'a pas de correspondance dans I'histoire de la littérature suisse en langue française. V., à ce sujet, Francillon, R. (dir) (1998), t. III, "Quelques aspects de la vie littéraire": 43-56 et IV, "La 'nouvelle littérature romande'": 31-57.

${ }^{11}$ Dont les articles de L'ère du soupçon furent publiés entre 1947 et 1956, et au dire de la romancière, "ont suivi de loin la parution de Tropismes" (Sarraute, 1956: [7]), instituant ainsi avec ce livre inaugural un dialogue non interrompu au fil des années. Nathalie Sarraute exclut son projet d'écriture, tel qu'elle l'affirme dans la Préface à L'ère du soupçon, de la séparation en vigueur entre "une littérature métaphysique, celle de Kafka [et celle] qu'on qualifiait avec dédain de 'psychologique"'; raison pour laquelle elle décide d'écrire l'essai "De Dostoïevski à Kafka" (Sarraute, 1956: 10).
} 
de laquelle, d'ordinaire, ces caractères se développent, ni sentiments connus et nommés" (Sarraute, 1956: 9). Lovay accentue l'écart du romancier de toute réalité située en dehors de celle de son texte même: chez lui pas de drames, pas de sentiments, même pas de sentiments inconnus ou non nommés, pas de psychologie. Sa quête se situe ailleurs, elle est tout aussi exigeante pour lui, romancier, que pour le lecteur de ses textes. Appuyée sur la défense intransigeante de sa liberté personnelle ${ }^{12}$, cette quête, poursuivie à chaque nouveau roman sur lequel Lovay travaille, est celle non pas de "commencer à raconter une autre histoire, mais de vivre l'histoire d'une autre écriture qui est la [sienne] en même temps qu'à chaque nouveau texte elle [lui] apparaît plus étrangère" ${ }^{13}$ (Lovay, 2004: 6).

De même le lecteur éprouve-t-il un sentiment d'étrangeté comparable devant les textes de Lovay. Des romans qu'il aurait du mal à "raconter", des romans qu'il ne saurait raconter, puisqu'ils déjouent ses habitudes de lecture du réel, lecture fondée sur des rapports de causalité entre les actions rapportées: "chez Lovay, le référent reflue devant un code autonome et travaillé en toute visibilité", considérait Jérôme Meizoz dans l'étude qu'il a consacrée à ce romancier en 1994, Le Toboggan des images, une étude particulièrement importante pour la connaissance de la "langue" lovaysienne (Meizoz, 1994: 59). Les romans de Jean-Marc Lovay mériteraient sans doute aussi la qualification de romans indécidables, au même titre que ceux analysés par Bruno Blanckeman dans l'étude qu'il publie en $2008^{14}$.

L'explication donnée par Lovay sur sa posture d'écrivain ${ }^{15}$ mériterait qu'on s'y attarde.

Pour Lovay:

1. Écrire, ce n'est pas "raconter une autre histoire";

2. Écrire, c'est "vivre" une autre histoire: celle de son écriture même;

3. Écrire, c'est se distancer de ce qu'on écrit, au risque de se confronter à une écriture qui n'est plus ressentie comme étant la sienne.

\footnotetext{
12 Dans la "Conférence de Stockholm" (1983), Lovay protestera contre la "violation de [son] espace aérien personnel", à la merci de satellites qui photographient les moindres incidents de sa vie quotidienne (Lovay, 1987: 11).

${ }^{13}$ C'est nous qui soulignons.

${ }^{14}$ Bruno Blanckeman associe étroitement la notion théorique de l' "indécidable" aux caractéristiques historiques de la contemporanéité et aux tendances esthétiques dont relève le récit littéraire français du dernier quart du XXe siècle (en particulier les récits de Jean Echenoz, Hervé Guibert et de Pascal Quignard): "À époque incertaine, récits indécidables" (Blanckeman, 2008: 11). L'auteur identifie les "opérateurs" qui soutiendraient ce concept selon trois lignes d'approche: "Fictions problématiques, par attraction et détraction indivises du romanesque; identités simultanément instituées et destituées, par des écritures autobiographiques inédites; langage à la fois ordonné et subordonné, dans des œuvres qui font de sa domination leur dynamique essentielle" (Blanckeman, 2008: 13). A l'exception de la tendance autobiographique, le roman Épître aux Martiens, bien qu'écrit à une période déterminante de la vie de Lovay, ce que le présent article se propose de démontrer, à l'appui de la correspondance entre Lovay et Maurice Chappaz citée, présente des caractéristiques d'écriture que ce concept met en valeur, tout en sauvegardant sa singularité. A remarquer d'autre part que les auteurs étudiés par Bruno Blanckeman "appartiennent [aussi] à la génération qui publia ses premiers textes dans les années 1970" (Blanckeman, 2008: 10).

${ }^{15}$ Nous nous permettons de revenir sur la citation, "non pas de commencer à raconter une autre histoire, mais de vivre l'histoire d'une autre écriture qui est la [sienne] en même temps qu'à chaque nouveau texte elle [lui] apparaît plus étrangère".
} 
Synthétisons-en quelques conséquences:

Le refus de la conception de la littérature comme "ayant quelque chose à dire", à partir d'une action qui progresse selon un rythme de cause-effet, fondée sur des catégories du récit "inventées"; le refus des récits référentiels, qui racontent une histoire et font appel à un pacte de lecture où les rôles (dont ceux du narrateur et du lecteur) sont bien déterminés par le récit et acceptés dès l'entrée en lecture.

Une intrigue semble pourtant être à l'œuvre dans chaque récit de Lovay. Dans le cas spécifique de l'Épître aux Martiens, un projet s'impose au héros-narrateur: celui de transposer le mur qui lui donnera accès à la ville de Tecnos, la ville Molosse, de travailler à la "révolution" avec des camarades. Néanmoins, les assises traditionnelles d'une "action"16 y sont bouleversées et les attentes du lecteur constamment déjouées. Tel que le constatait Jérôme Meizoz, dans l'étude tantôt référée, ses textes, privilégiant la "digression" et poussant le lecteur "sur des fausses pistes", empêchent "toute lecture globalisante" (Meizoz, 1994: 52). Pour Meizoz, les récits de Lovay se situent "aux antipodes du réalisme".

Refusant au récit la fonction de "raconter", Lovay lui en attribue une autre, puisqu'il ne cesse d'intituler ses textes comme des "romans" (donc des textes fictionnels): celle de se dire eux-mêmes en tant que récits, en éludant toute possibilité référentielle. Un récit certes "auto-représenté", "laboratoire", dont la prise de conscience a été très tôt assumée par le romancier sur le mode de la dérision tragique. C'est en ces termes qu'il s'est exprimé dans la "Conférence de Stockholm" (incluse dans le livre Conférences aux Antipodes, 1987), une conférence qu'il prononce lors d' "une soirée littéraire à la Bibliothèque Nobel le 14 septembre $1983\left[{ }^{17}\right]$ devant une assistance très nombreuse du monde des lettres et des notables": "Je ne savais pas encore que écrire n'est pas décrire. Je découvrais qu'il n'y aurait jamais de fin à aucune description" (Lovay, 1987: 12).

Lovay parvient ainsi à bouleverser les fonctions et les catégories traditionnelles du roman - dont celle d'auctorialité comme entité légitimatrice du texte et de son interprétation , tout en disloquant son centre d'intérêt vers le récit lui-même, celui-ci l'intéressant en tant que romancier comme son domaine de travail ("laboratoire narratif", selon Jérôme Meizoz (1994: 145)), et vers le destinataire de ce récit, le lecteur. Meizoz l'avait déjà signalé: chaque texte de Lovay se donne à lire comme "résistance à l'explication" (id.: 146).

Notre hypothèse se confirme, il nous semble alors légitime de considérer que le lecteur et ses habitudes de lecture sont le principal destinataire des récits de Lovay. Ce que le titre du roman semble par ailleurs confirmer: une épître, c'est une lettre adressée à des disciples, mais aussi à tout un peuple qu'il faut conduire dans la bonne voie, par l'exemple; une épître, c'est aussi un texte sacré, un texte qui légitime une parole. Par conséquent, un

\footnotetext{
${ }_{17}^{16}$ Dont le modèle narratologique a été étudié par G. Genette dans le livre si connu, Figures III, Paris, Seuil, 1972.

17 L'année où le prix Nobel de la littérature fut attribué à l'écrivain britannique William Golding.
} 
texte exemplaire, véritable "art poétique" adressé à un lecteur à qui il faudra apprendre à lire autrement, qu'il faudra conduire dans de nouveaux sentiers, à qui il faudra faire perdre les mauvaises habitudes de lecture....mais qui a encore besoin d'un guide: l'Épître est bien le premier livre de toute la production à venir...

Pourtant, une épître adressée à des Martiens. À des lecteurs nouvel arrivés dans le pays inconnu d'une littérature neuve? Neuve pour celui qui l'écrit, tout autant que pour celui qui va la lire? Livre d'apprentissage, voire initiatique, et à double titre, pour l'auteur et pour son lecteur?

L'auteur s'exprime lui-même sur ce roman dans quelques lettres adressées à Maurice Chappaz lors de son voyage en Afghanistan entre 1968-1970, Chappaz étant un écrivain qu'il admire et à qui il enverra l'Épître, de New Dehli.

Tel que l'on peut lire dans la postface à cette correspondance, signée par Jérôme Meizoz, qui l'intitule avec propriété, "Le moment Rimbaud", les "premiers textes connus [de Jean-Marc Lovay] sont contemporains de ces voyages en Asie, d'Istanbul en Afghanistan, puis au Népal. Lovay rédige alors l'Epître aux Martiens' qu'il envoie à l'aîné de New Dehli" (Chappaz; Lovay, [1970]: [135]).

Quelques références à l'Épître aux Martiens sont repérables dans la correspondance entre l'écrivain consacré et le jeune voyageur-écrivain. Lisons-le dans la lettre que celui-ci lui écrit en Afghanistan le 15 août 1968. Une lettre dense, où le voyageur transmet à son interlocuteur tout son désir de se libérer de toute trace de ses origines occidentales. II se livre alors à un dur procès de désappropriation de soi, au rythme de sa traversée des montagnes afghanes. Un procès qui passe par la destruction délibérée de toute trace physique de cette appartenance, à commencer par celle de son "vêtement européen", qu'il "[foule] aux pieds“, et qu'il confronte à sa "pourriture spirituelle". La conscience de celle-ci lui devient insupportable face à la "désolation du monde" qu'il constate au long de son expérience orientale, et que d'autres jeunes que lui partagent à leur tour, parcourant eux aussi la même Route. Le lecteur comprendra alors la raison pour laquelle, à peine écrit, ce livre semble avoir perdu tout son sens pour son auteur: "Mon premier roman qui fait le mort dans un buffet suisse, il s'intitule 'Epître aux Martiens', une histoire de soleil qu'un type voudrait revoir au moins une fois, une seule, dans une cité de béton. Cela c'est fini" (id.: 33).

L'attention portée au sentiment que le voyageur éprouve devant la "désolation du monde" - dont il se laisse enivrer ${ }^{18}$ - fera mieux comprendre au lecteur la raison pour laquelle l'objectif premier de ce livre lui apparaît désormais révolu: "Les épîtres, je m'en fous. II me semble ici que je ne veux surtout rien dire aux autres" (id.: 33).

\footnotetext{
18 "toute cette enivrante désolation du monde" (id.: 32).
} 
Plus loin, dans la même lettre, il insistera sur le sens que prend pour lui l'expérience de la Route. Rejetant "tout l'exotisme d'artifice de l'Afghanistan", et méprisant les "nouveaux civilisés orientaux de Kaboul, de Téhéran, de Delhi", devenus les "caricatures" des occidentaux, sa "route" lui apparaît dans toute la beauté de son "inutilité". II en fait l'aveu en s'adressant à Chappaz en ces termes: "Savez-vous que ma route apparaît si belle d'inutilité!" (id.: 36).

Et pourtant, ce livre, il y tient et il ne cesse d'y travailler en vue de sa soumission prochaine pour publication, en pleine conscience déjà de son identité de romancier. La référence est claire au genre que Lovay attribue au livre dont il est l'auteur, dans la lettre citée, le premier qu'il ait écrit: "Mon premier roman..... Un romancier prend conscience de soi, et un projet d'écriture s'annonce en germe, à lire ce passage.

Lovay reviendra sur ce livre dans les lettres suivantes de cette correspondance.

De Katmandou, déjà au Népal, il s'adresse le 8 avril 1969 à Maurice Chappaz, en faisant toute confiance à celui-ci pour la lecture de son manuscrit, et en lui attribuant toute autorité pour détruire les passages que le poète jugerait "superflus, ou d'une complication trop ésotérique" (id.: 41). Tel qu'il le reconnaît, en termes personnels, "cet Épître aux Martiens explique ma Route et d'autres routes, et rien de plus concret que ce chemin de privations, d'éclairs, et de fumeries au soir, quand on sent grandir l'errance..." (id.: 41); la portée sociale de ce livre est pourtant voulue d'une plus grande ampleur par le romancier et ses semblables (les "beats français" ou les "vagabonds rencontrés en Afghanistan, à Karachi [...] ailleurs" (id.: 42)), qui insiste sur sa haine de la société européenne en ces termes: "Oui, l'Epître est un carnet jeté sur la table occidentale: la justification d'une existence, d'existences de rejet et d'insulte, et de nos bonheurs arrachés à la Machine, une accusation!“" (id.: 42-43).

La réponse de Chappaz ne se fera pas attendre: un dialogue de poètes s'établit entre les deux hommes. Le 16 avril 1969 il lui adresse de Veyras, dans le Valais, l'une des lettres les plus importantes de cette correspondance, tant du point de vue humain (Chappaz est sans doute la voix que Lovay respecte, il s'adresse à lui par "Monsieur Chappaz" tandis que le poète le tutoie, l'aîné qui le sent courir le risque de s'égarer complètement dans la Route), que du point de vue littéraire.

Nous nous permettons d'en extraire un long passage:

Le silence d'une montagne me pénètre et l'espace d'une nuit qui tremble.

Franchement malgré les mers je ne me sens pas loin de toi. Si je devais te donner un conseil je te dirai:

Intériorise.

Ne fume que comme on boit un verre de vin (le 'glacier' d'Anniviers). 
L'imagination vraie est celle qui nous permet de créer. L'écriture est un rêve assumé. Sois farouchement sincère: critique-toi par rapport à ce que tu écris jusqu'à ce que tu éprouves vraiment la nécessité de chaque substantif, chaque adjectif. Aucun emprunt. Aucun théâtre. II y a la vie qui nous échappe à chaque instant ${ }^{19}$. On peut se donner le change avec des mots comme on flatte un ivrogne qui va mourir. Et tout l'univers est présent dans le suspens des haï-kaï à la plus fine pointe de l'attention.

Tout à fait silencieuse

Et brûlante de désir

La luciole

Plus digne de pitié

Qu'aucun autre insecte (id.: 49-50).

L'entente profonde entre les deux écrivains voyageurs s'exprime par l'usage d'un vocabulaire dont le symbolisme est perçu des deux. C'est ainsi que les mots de "hippie" et de "poète" deviennent synonymes dans leur correspondance. "Le seul vraie hippie est le poète. Et il est aussi une luciole", lui écrira le poète qui vient de publier Match Valais-Judée, le violent manifeste dans lequel Lovay, malgré la distance considérable qui le sépare de son pays, reconnaît déjà "ce mach Vie normale contre Surproduction, Surexcitation des producteurs et des consommateurs, des machiavéliques puritains" (id.: 40), proposant par ces mots un parcours de lecture comparée des deux livres. L'affinité se resserre entre les deux poètes. Écoutons encore Chappaz dans la lettre citée: "Cher Jean-Marc, je parle poésie: tu as du tempérament, tu es viril, crée en toi une ardente absence" (id.: 50); plus loin il fera comprendre au jeune poète la source de toute poésie: "ma source est en moi" (id.: 52).

Cette correspondance nous révèle ainsi l'entente profonde entre deux hommes que plus de trente ans séparent, Chappaz étant né en 1916 et Lovay en 1949. Une entente sans doute soudée par l'amour partagé pour le Valais, duquel ils sont tous deux originaires, et dont la présence mystique nourrit l'œuvre. L'assimilation du Valais au Tibet, par Maurice Chappaz dans son livre Valais-Tibet: Icône des pays de montagne, publié l'an 2000 à l'Atelier du Cadratin, à Le Châble, Vevey, acquiert toute sa signification dans le contexte de cet amour et d'une aventure de vagabondage qui donna l'occasion au voyageur de dénoncer la destruction de "'origine des origines" (Chappaz, 2000: 13), de "plusieurs civilisations" que l'homme n'a fait que 'secouer', comme s'il [fracassait] "de vieux pruniers" (id.:12), sans se rendre compte du caractère irréversible des "grandes cassures" qu'il a précipitées (id.: 11). Chappaz parcourt le monde depuis sa jeunesse, il se retrouvera dans l'Himalaya, "marchand à l'aube, [...] dans l'extrême automne, en 1970, dans la vallée de la Kali Gandaki", peu après le voyage de Lovay en Asie. Le souvenir du "murmure" qu'il entend au loin, en apercevant

\footnotetext{
${ }^{19}$ En italique dans le texte.
} 
une lointaine "lignée de silhouettes" affleurera à son esprit trente années plus tard, lorsque, rentré définitivement chez lui, il prend conscience de l'impossibilité de nouveaux voyages: "Si je songe au retour au Châble, d'où je ne bougerai plus..." (Chappaz, 2000: 58).

Grands voyageurs, les années soixante du siècle dernier les font se rejoindre dans le même esprit de révolte et de vagabondage qui traverse l'Europe à l'époque: Lovay qui décide d'abandonner ses études à seize ans et demi, et qui écrit dans un premier article, "Collège et Montagne": "le tableau noir paraissait blanc, tant la noirceur du maître frisait l'excès"20 (Chappaz; Lovay [1970: [134]), et Chappaz qui le défie à partir avec lui et Bertil Galland en Laponie ${ }^{21}$, "à la Saint-Jean fêter le passage hiver-été [...] au-delà le cercle polaire, et partager le désir de s' "enfoncer dans la grande nuit qui dure vingt-quatre heures sur vingt-quatre, et en sortir tout doucement accouché par la grande débâcle d'avril. Comme les bourgeons des saules" (Chappaz; Lovay [1970]: 53).

Or, pourquoi ce désir d'errance, cette mobilité constante chez les deux hommes?

La lecture de la correspondance qu'ils entretiennent à l'époque nous en apporte quelques réponses. Des réponses qui nous permettent de mieux lire l'Épître aux Martiens, d'une part et, d'autre part, de comprendre à quel point leur quête d'authenticité les réunit, de comprendre à quel point leur conscience écologique sous-tend leur œuvre et leur vie. A la différence près que si chez Chappaz la dénonciation est publique ${ }^{22}$, ou assume des formes d'expression lyrique particulièrement sensibles dans ses derniers textes, dont le livre cité (Valais-Tibet: Icône des pays de montagne) constitue une référence incontournable dans le parcours de l'engagement de l'auteur, chez Lovay elle émane de la substance de ses écrits. S'assumant comme thème dominant de l'Épître, la dénonciation vise dans ce livre des aspects aussi visibles et récurrents que la Surproduction ou la ville de Tecnos, les méfaits du dollar, la surveillance policière, que le romancier énumère: "ma haine de la Surproduction, de Tecnos, des livides truands du dollar, de la fausse barbe policière" (Chappaz; Lovay [1970]: 57). La nuit de la Laponie devrait accomplir le dessein purificateur souhaité: "Une nuit comme si l'éclipse de l'Epître aux Martiens s'abattait sur leur charogne de société" que Lovay décrit dans la lettre qu'il adresse à Chappaz de Katmandou, le 5 mai 1969 (id.: 60).

La lecture de Valais-Tibet concourt également au tracé d'un itinéraire purificateur. Long poème en prose, méditation à l'instar de celle entendue dans l'Himalaya qui s'est prolongée "sans discontinuer comme le vent" (Chappaz, 2000: 59) au long de la vie du poète, ce livre clôt le périple qui fit rencontrer deux êtres en quête d'authenticité, partis à la recherche du "regard de l'inculte que nulle soupe culturelle n'a délavé" (Chappaz;

\footnotetext{
${ }^{20}$ Passage transcrit dans la postface écrite par Jérôme Meizoz à l'édition de la correspondance citée.

${ }^{21}$ V. Lettre du 16 avril 1969.

22 V. L'engagement civique du poète lors de sa participation aux travaux du barrage, ses articles dans des journaux; ses attaques aux entrepreneurs, et des publications telles que Le Match Valais-Judée (ou Les Maquereaux des cimes blanches).
} 
Lovay [1970]: 82), décidés à retrouver le "primitif" dans les contrées les plus éloignées, ayant toujours le Valais, un Valais désormais perdu, comme toile de fond à leurs mémoires. Nostalgie d'un monde révolu où, pour Chappaz "la coupure avec le sacré, l'invisible, s'apercevait à peine" (Chappaz, 2000: 26) dans le rythme de la vie paysanne du Valais de sa jeunesse; quête d'un monde possible où, pour Lovay, peut-être existe-t-il encore, à l'époque où il écrit à Chappaz, la possibilité du salut, dans la poursuite d'un périple qui est avant tout celui de la libération de soi. Ce dont le jeune romancier se rendra compte au long du voyage, tel qu'en témoigne l'épisode de la rencontre avec une prêtresse dans un petit temple au Népal, qui lui fera "assassiner une théorie existentialiste que toujours [il crut] indestructible", expérience qu'il décrit à son interlocuteur dans sa lettre du 5 mai 1969 écrite à Katmandou (Chappaz; Lovay [1970]: 61-62). Un voyage qui le mettra à nu devant des "absurdités européennes", lui fera prendre conscience de ses "vaines années de verbiage perdu" (Chappaz; Lovay [1970]: 79), tel qu'il avouera à son retour, dans une lettre datée du Valais, en juin 1969. Un voyage qui le mettra à nu devant lui-même, devant lui permettre d'aboutir "à l'unité", "vidé de [lui-même], comme s'il était un personnage fait de mille couches superposées de carton" (Chappaz; Lovay [1970]: 83), de par sa seule "lumière intérieure". Lovay atteindra alors la "communion" totale avec l'univers, la "communion avec le global qui ne s'exprime point par la conquête", communion qu'il avait déjà désirée à Kaboul, en ce mois de juin 1969 (Chappaz; Lovay [1970]: 26).

La dénonciation exige chez cet auteur des formes d'expression propres, qui soutiennent un univers fictionnel dont l'Epître aux Martiens révèle un tableau dévastateur. Ce livre ouvre ainsi l'espace à l'expression de ce "primitif' en circulation libre" que Maurice Chappaz reconnaissait au groupe d'amis de Lovay, et leur "aptitude à le transformer", dans une lettre datée des 9-10 juillet 1969, expédiée de la Laponie (Chappaz; Lovay [1970]: 93). La quête de l'expression du "primitif" justifiera pour Lovay, imbu de philosophie bouddhique, l'expérience d'une "oasis où plus rien ne trouble" (Chappaz; Lovay [1970]: 61).

C'est justement de "circulation libre" qu'il s'agira dans Epître aux Martiens, "circulation libre" de la parole romanesque, des situations fictionnelles, de la composition du récit. Refusant l'écriture réaliste - fondée sur l'illusion du réel - c'est bien au voyage que Lovay nous invite, un voyage traduit par le déplacement des mots et de la syntaxe, davantage qu'aux voyages des personnages, eux-mêmes surpris par l'imprévisibilité des situations qu'ils ne maîtrisent plus, ayant perdu toute attache avec un passé romanesque dans lequel le lecteur leur reconnaissait des portraits fiables et les identifiait grâce à des référents sécurisants. Refusant la présentation des événements selon un ordre chronologique progressif et leur enchaînement d'après les règles de la causalité, le récit lovaysien institue une temporalité autre, où les limites entre le passé, le présent et l'avenir deviennent difficilement localisables. L'inscription des événements sur un temps qui ne peut être autre 
que celui de leur lecture, fait étrangement coexister sur un même espace plusieurs temporalités, tel que ce passage l'illustre:

Et maintenant il marchait, ne sachant plus sa vie passée, mais conscient d'outretombe, au-delà des amis perdus, de la vie vaste et passée, mais conscient au-delà de tout, d'une décision, de sa décision si dangereuse et concrète, si vengeresse, et les voluptés le brimaient lui en brimant la banlieue, et il se demanda quand l'éclipse surgirait, mais cela étant loin dans le temps, et la lune masquerait l'astre vocalement, comme un poisson qui gobe une mouche, et les pleurs des fous glaceraient les lumières entre les végétaux, juste avant les crépuscules, les belles années éperdues (id.: 18).

Si le récit de Lovay bouleverse la perception traditionnelle du temps, en tant que catégorie narrative, puisqu'il n'a d'autre existence que, d'une part, celle de l'expérience même qu'en font les personnages au moment où ils la font et, d'autre part, celle de l'expérience de lecture au moment où celle-ci s'accomplit, l'espace évoqué dans ses romans est transfiguré par le sens que les personnages, ou le narrateur, lui accordent. C'est ainsi que l'un des personnages de l'Épître aux Martiens, Julot, pense "à la démâture de l'Axe terrestre, et [que, pour lui] la Terre [bascule] sur l'Axe inversant les Pôles" (Lovay, 2004: 16), ou que, décrivant le trajet que fait le camion où est entré Julot et, un peu plus tard, trois autostoppeurs, le narrateur reconnaît que "la plaine n'était comparable à aucune plaine de l'existence de Julot", établissant ainsi une démarcation claire entre les référents connus du personnage (et du lecteur) et des référents que le récit construit: "La plaine, c'était l'image que certains humains se firent parfois du vide. C'était cela, cette plaine, une image du vide comme certains humains en trafiquèrent dans les cabarets de la science ou des cultures, mais le vide n'a pas de bords profonds comme ceux croisés tout au long du trajet" (id.: 21).

L'exercice de déconstruction des assises traditionnelles du roman dont témoigne ce livre constitue la première forme de manifestation pour Lovay, sur le plan littéraire, du désir de libération de toute trace de culture européenne exprimé par le romancier au long de la correspondance qu'il allait bientôt échanger avec Maurice Chappaz. La lecture croisée du roman et de la correspondance s'avère donc, à notre sens, fondamentale pour la compréhension de l'univers de Lovay, et de la poursuite par celui-ci d'une route existentielle mais aussi esthétique, nouvelle. Davantage que la réunion de documents susceptibles d'intéresser le seul biographe, cette correspondance se donne aussi à lire comme l'espace de la réflexion métalittéraire de l'écrivain.

L'expérience de l'affranchissement total de soi et de l'écriture romanesque est dite par le personnage Julot, qui se déploie dans un torrent de langage que rien ne semble 
pouvoir arrêter: à la vitesse de son énonciation correspond l'intensité des verbes dont il fait un usage multiplicatif en quête d'un temps "Antérieur", paradoxalement "magique et véritable". Le passage suivant en accentue l'agressivité sémantique et la pulsion de mort:

II avait bu avec passion, pour devenir lucide, pour noyer, enivrer, amoindrir, dévergonder et perclure toutes les philosophies déposées en son cerveau par des puissantes morbides. Abêtir les paroles tapies en soi et fermentant, les démystifier, abuser d'elles, surpasser enfin l'essence de leurs dépôts, les admirer comme on admire les végétaux, les feux de brousse, les corps béatifiés dans les écuries impossibles de l'Antérieur véritable, magique et véritable! (Lovay, 2004: 17).

La répétition de l'adjectif "véritable", qui caractérise une antériorité située, à son tour, dans un espace "impossible", autoriserait éventuellement le lecteur à considérer ce temps comme le temps unique et singulier. Un temps où "philosophie" et "parole" se répondraient, où leur unicité et singularité étaient encore possibles, pas encore dénaturées dans des proliférations plurielles ("les philosophies", "les paroles").

Le récit lovaysien demande au lecteur une nouvelle posture. II lui demande de se rendre disponible pour accepter d'assister au déroulement des images que le langage lui offre, en dehors de toute finalité de représentation du réel, dans le refus de tout modèle préexistant (fût-il philosophique) d'appréhension de ce réel. Un réel référentiel (aussitôt démystifié par un récit qui déjoue les repères spatio-temporelles auxquelles s'attendrait le lecteur), ou un réel qui correspondrait à la prise de conscience approfondie de soi (aussitôt déjoué par les dialogues inconséquents qui traversent le récit). Ce récit s'écarte ainsi de toute tendance introspective qui caractérisait l'écriture de maints nouveaux-romanciers français contemporains de Lovay, particulièrement intéressés par les différentes modalités du monologue intérieur, que ces modalités illustrent le jaillissement de la parole empêchée chez Sarraute, ou le mot-thème chez Claude Simon. Chez Lovay, le récit s'auto-génère, sans d'autre motivation que celle-là même de sa propre corporisation, dans "un code autonome travaillé en toute visibilité", tel que l'a constaté Jérôme Meizoz (Meizoz, 1994: 59) ${ }^{23}$, en recourant à l'expression qu'il cite de Jean Ricardou (id.: 56, n. 46). Dans un univers où les repères temporels traditionnels ne font plus sens, Verdi, l'un des personnages, s'écrie "- Quelle grande question est éternelle?..." (Lovay, 2004: 74).

La quête d'un au-delà, temporellement et spatialement non localisable, soutient l'action romanesque de l'Épître aux Martiens. C'est sans doute le but du déplacement, hâtif, sinon désespéré puisque inéluctable, du héros-narrateur du roman, Julot, décidé à transposer un mur situé au bout de l'immense plaine référée plus haut. La prise en compte

\footnotetext{
${ }^{23}$ Citation déjà référée dans les premières pages de notre étude.
} 
seule de cette "décision" aux conséquences imprévisibles justifie son action, une action à laquelle il se prépare, telle épreuve initiatique pour passer dans ce "là-bas" (id.: 14) invoqué par l'un des personnages: "Il y a des instants où je ne sais plus si j'ai vraiment tout tué en moi! C'est une grave décision..." (id.: 12).

Ecoutons encore une fois Chappaz (lettre du 19 mai 1969, Veyras), qui parle du livre de Lovay à ses "compagnons", tout en accentuant son "actualité mythique": "Le livre de Lovay avec sa verbosité naïve, lyrique, sa confusion précise, son crayonnage d'une fumée où les personnages se découvrent comme des signes, eh bien c'est le torchon qui brûle": 75). La portée politique des lettres échangées entre les deux épistolographes, de même que celle de l'Épître aux Martiens est clairement énoncée par Maurice Chappaz dans la même lettre, à la tonalité prémonitoire. Pour le poète du Valais aussi,

notre présent et notre avenir [...] bourronnent au coin du bois. On en connaîtra des crises! La civilisation industrielle va vers la guerre. On se défendra avec de l'irrationnel dans tous les domaines $[\ldots]$

II y a quelque chose de vague dans ton livre qui peut être une faiblesse et que j'aime comme j'ai aimé la fausse révolution pratique du mois de mai.

Un mois de mai plus réel qu'ils ne s'en doutent. (Chappaz; Lovay [1970]: 75)

Proches l'un de l'autre, "malgré les mers" (Chappaz; Lovay [1970]: 49), tel que le reconnaît Chappaz, qui lui écrivait de Veyras le 16 avril 1969, les deux hommes anticipent sur la condamnation du mode de vie occidental. Cette condamnation anéantira prévisiblement aussi les contrées où d'aucuns cherchent encore la plénitude (comme les deux pèlerins de Birmanie, les yeux rouges de cannabis qui poursuivaient leur route dans leur quête d'ascètes), que ce soit le Valais (dont les quelques contrées primitives signalées par Chappaz seront bientôt condamnées), la Laponie (vers laquelle Chappaz projette d'aller avec son ami, l'éditeur Bertil Galland, d'ascendance suédoise), ou l'Inde, pays d'où vient de partir Lovay, en route pour le Népal.

Intéressé par les enseignements du Boudha - qui deviennent pour lui sa "façon de fumer un peu l'Orient" (Chappaz; Lovay [1970]: 48) -, Chappaz partage un profond désir d'ascèse à laquelle Lovay répond par son expérience personnelle de la Route, et par son observation aiguë des autres, "pèlerins" comme lui dans ce viatique.

Expérience de libération, le voyage et l'écriture permettent à Lovay de se vider de soi, afin de garder sa vision lucide du réel. Pour les deux, c'est cela que d'être poète; c'est cela que les deux attendaient de leur errance incessante, dans la quête de régions encore non atteintes par la "soupe culturelle", en pleine conscience de la catastrophe éminente. Or, cette expérience du monde exigeait une manière pour le dire. La vision apocalyptique sera 
récurrente dans les romans futurs de Lovay. Les scénarios de chaos, la vision d'une humanité perdue qui ne garde que des mémoires lointaines d'un temps que le lecteur aurait du mal à concevoir comme révolu ou comme futur remplissent ses pages de passages à la limite du lisible ${ }^{24}$, mais combien clairvoyants, d'un monde postcivilisationnel ${ }^{25}$ :

Le camion s'enfonçait dans la plaine, et des morceaux de vide glissaient sur le capot, s'enfilaient dans la cabine, tandis qu'un silence dur et immobile s'emparait de l'espace. Et la plaine était sans arbres, sans rivières, sans herbes inoubliables agitées par la brise. Des murs de boue figée se dressaient et retombaient, fixés par les yeux du temps et les regards humains caillés dans les interstices du camion, avec dans l'horizon, dans l'unique horizon, l'image faussement sidérale du vide, imprégnant les argiles de cette plaine nouvelle. Nulle part construction humaine, point de vie cellulaire dans les trous saumâtres de la piste (Lovay, 2004: 24).

L'acheminement vers cet univers est déjà pressenti dans les échanges épistoliers auxquels nous nous sommes référée: "On a médité sur la fin du monde: c'est la civilisation qui se vomira doucement sans le Spasme Atomique" prévoit Lovay (Chappaz; Lovay [1970]: 80). Cet acheminement acquiert dans l'Épître le caractère d'urgence: la catastrophe est alors envisagée comme "une crise, une agonie où se trouve encore la seule chance" par Chappaz, qui partage ce sentiment avec son interlocuteur, tel qu'il le lui avoue dans la lettre qu'il lui adresse le 16 avril 1969: "Je t'écris cela parce qu'en profondeur, intérieurement, je voudrais échapper à cet Occident qui a bouffé le Valais, qui mangera l'Inde qui est deux ou trois mille fois plus grande (mais qui certes se désagrègera dans une crise, une agonie où se trouve encore la seule chance). Le 'Moi' de l'Occidental mange aussi sa conscience. La retrouver, faire vivre (naître d'abord) en soi une liberté n'est pas facile", affirme Maurice Chappaz dans la lettre citée (id.: 48). Propos auxquels Lovay répondra, dans la lettre du 5 mai 1969, expédiée de Katmandou: "Vous dites que l'Occident bouffera l'Inde; c'est commencé. On voit des charpies sanglantes sur les routes, même à Bénarès: l'immonde épouvantail du Coca-Cola..." (id.: 56).

La catastrophe à laquelle font appel un roman tel que l'Épître aux Martiens et la correspondance qui le suit de peu devient une nécessité, à caractère urgent, sous la plume des deux auteurs. Elle découle d'une exigence: celle de la dénonciation - par le romanesque, par la poésie - de la cassure de l'Occident. Dénonciation déclenchée par

\footnotetext{
24 “Indécidables", par leur singularité dans le contexte des lettres romandes ou françaises contemporaines.

25 Nous empruntons l'expression à Doris Jakubec, dans l'article publié dans la Revue des Belles Lettres, décembre 2009, à propos de la parution du roman de Lovay, Tout là-bas avec Capolino. Nous remercions Doris Jakubec de nous avoir communiqué le manuscrit de son article avant publication. La vision d'un univers postcivilisationnel constituait déjà l'un des thèmes majeurs d'Épître aux Martiens, que le romancier poursuivra dans son œuvre future.
} 
l'Epître et auquel des romans postérieurs de l'auteur accorderont d'autres tonalités. Ce passage de la lettre adressée le 5 mai 1969 de Katmandou à Chappaz l'introduisait déjà: "II faudra miser sur une catastrophe, une agonie", puisque "ce qui importe, c'est ce qui le motiva", "l'instrument n'a aucune importance" (Chappaz; Lovay, [1970]: 57). Le roman énoncera clairement cette motivation et en fera le noyau de son intrigue: la dénonciation, la révolte, la "révolution", mot-clé de l'Epître aux Martiens. Ceci expliquerait sans doute pourquoi chez Lovay, les humains ressemblent à des androïdes. Les Martiens, on le comprendra, ce sont les androïdes qu'il côtoie, qu'il peut retrouver soit chez-soi, soit en Inde, un peu partout dans le monde déjà globalisé, celui de la Surproduction et de la ville de Tecnos, un monde qu'il a "dû réapprendre à côtoyer", tel qu'il l'écrit à son retour au Valais, en juin 1969 (Chappaz; Lovay, [1970]: 77). Les Martiens sont sans doute ceux-là mêmes qui le rejettent dans leur intolérance et leur cupidité, ceux-là mêmes dont les agressions contre la planète sont dénoncées par les deux poètes, sans doute aussi, les lecteurs à qui il faudra apprendre à lire les romans et le monde, autrement.

Tel que le mot "ouverture" cité plus haut ${ }^{26}$ le laissait supposer, chaque livre de Lovay nous convie à un mode particulier de lecture. Davantage qu'à l'étude des possibilités de dysfonctionnement des catégories formelles du récit comme motif du récit même (ce qui constitue pourtant l'occasion de la reconnaissance du travail formel exigeant élaboré par le romancier, mais ne pourrait épuiser la lecture de l'œuvre littéraire), les récits de Jean-Marc Lovay nous invitent à partager avec l'auteur une conscience aiguë du monde contemporain. Une conscience que le roman semble plus que jamais le genre approprié à partager avec ses contemporains. C'est sans doute dans la mesure où Lovay construit ses romans sur ce dysfonctionnement formel - qui demande d'autres outils de lecture que ceux fournis par tout système d'approche rassurant -, que ses romans se donnent à lire comme des "formes planétaires", pour utiliser la formule proposée par Franco Moretti pour caractériser la souplesse qu'il constate au roman au long de son histoire millénaire ${ }^{27}$, une souplesse qui est aussi le garant de sa survie.

\footnotetext{
${ }^{26} \mathrm{~V}$. les premières pages de cette étude.

${ }^{27}$ Franco Moretti la reconnaît en ces termes: "The novel as culture [...], but certainly also as form, or rather forms, plural [...] as the borders of literature are continuously, unpredictably expanded [...] But thanks to it, the novel becomes the first truly planetary form: a phoenix always ready to take flight in a new direction, and to find the right language for the next generation of readers" (Moretti, 2006: [ix]).
} 


\section{Bibliographie}

BalzaC, Honoré de (1989). Le Père Goriot, Paris, Presses Pocket.

BLANCKEMAN, Bruno (2008). Les récits indécidables: Jean Echenoz, Hervé Guibert, Pascal Quignard, Paris, Presses Universitaires du Septentrion.

ChAPPAZ, Maurice ; LovAY, Jean-Marc [1970]. La Tentation de l'Orient, Carouge-Genève, ZOÉ-Poche.

CHAPPAZ, Maurice (2000). Valais-Tibet : Icône des pays de montagne, Le Châble, Vevey, Le Cadratin.

LovAY, Jean-Marc (1987). Conférences aux Antipodes, Genève, Éditions Zoé.

LovAY, Jean-Marc (2004). Épître aux Martiens, Carouge-Genève, Éditions Zoé.

MEIzoz, Jérôme (1994), Le Toboggan des images: Lecture de Jean-Marc Lovay, Carouge-Genève, Éditions Zoé, coll. Critique.

MoretTI, Franco (2005) (ed.). The Novel, Princeton and Oxford, Princeton University Press, vol. I, "History, Geography, and Culture". 